\title{
Educação continuada e metodologias ativas em cursos a distância em enfermagem: revisão integrativa da literatura
}

RESUMO | Objetivo: Verificar e discutir as metodologias ativas de ensino adotadas na educação de enfermeiros em cursos à distância. Método: A pesquisa foi aprovada pelo Comitê de Ética em Pesquisa da Universidade Federal de São Paulo. Realizou-se revisão integrativa da literatura em bases de dados utilizando os seguintes descritores e suas combinações: Educação a Distância, Enfermagem e Metodologias ativas. Resultados: Observou-se que 33,3\% dos estudos promoviam aprendizagem por meio de ambiente virtual de aprendizagem, 30,3\% usavam o formato e-learning e 12,1\% utilizavam método híbrido. Considerações finais: As metodologias ativas de ensino têm sido empregadas em cursos de capacitação de profissionais de saúde, inclusive enfermagem, e têm contribuído positivamente na qualidade da assistência prestada aos pacientes.

Palavras-chaves: Educação em Enfermagem; Enfermagem; Educação à Distância; Aprendizagem; Aprendizado Ativo.

\begin{abstract}
Objective: To verify and discuss the active teaching methodologies adopted in the education of nurses in distance learning courses. Method: The research was approved by the Research Ethics Committee of the Federal University of São Paulo. An integrative literature review was carried out on databases using the following descriptors and their combinations: Distance Education, Nursing and Active Methodologies. Results: It was observed that 33,3\% of the studies promoted learning through a virtual learning environment, 30,3\% used the e-learning format and $12.1 \%$ used a hybrid method. Conclusion: Active teaching methodologies have been used in training courses for health professionals, including nursing, and have contributed positively to the quality of care provided to patients.
\end{abstract}

Keywords: Nursing Education; Nursing; Distance Education; Learning; Active Learning.

RESUMEN | Objetivo: Verificar y discutir las metodologías activas de enseñanza utilizadas en los cursos de educación a distancia para la educación en enfermería. Métodos: El estudio fue aprobado por el Comité de Ética en Investigación de la Universidad Federal de São Paulo. Se realizó una revisión bibliográfica integradora utilizando los siguientes descriptores y sus combinaciones: educación a distancia, enfermería y metodologías activas. Resultados: Se observó que el 33,3\% de los estudios promovió el aprendizaje a través del entorno de aprendizaje virtual, el 30,3\% utilizó el formato de aprendizaje electrónico y el 12,1\% utilizó el método híbrido. Consideraciones finales: Se han empleado metodologías de Aprendizaje Activo en cursos de capacitación para profesionales de la salud, incluida la enfermería, que han contribuido positivamente a la calidad de la atención brindada a los pacientes.

Palabras claves: Educación em Enfermería; Enfermería; Educación a Distancia; Aprendizaje; Aprendizaje Activo.

\section{Flávia Carla Takaki Cavichioli}

Enfermeira. Mestre em Ciência, Tecnologia e Gestão Aplicadas a Regeneração Tecidual pela UNIFESP.

ORCID: 0000-0003-2277-9548

\section{Hélio Martins do Nascimento Filho}

Enfermeiro. Mestre em Ciência, Tecnologia e Gestão Aplicadas à Regeneração Tecidual pela Unifesp. Enfermeiro na Unidade Saúde da Família / USF em Conselheiro Lafaiete - MG. Professor na Pós-graduação em Enfermagem Dermatológica com Ênfase no Tratamento de Feridas na Faculdade FAIPE (Cuiabá-MT).

ORCID: 0000-0003-3700-3910.

\section{Daniela Tinti Moreira Borges}

Enfermeira. Mestre Profissional em Ciências pela UNIFESP/SP; Estomaterapeuta, pela FAMERP/SP (2019). Docente de Graduação em Enfermagem na AEMS (Faculdades Integradas de Três Lagoas/MS).

ORCID: 0000-0001-8890-8918

\section{Leila Blanes}

Enfermeira. Doutorado em Ciências pelo Programa de Pós-Graduação em Cirurgia Plástica da Universidade Federal de São Paulo. Vice-coordenadora e Orientadora do Mestrado Profissional em Ciência, Tecnologia e Gestão aplicadas à Regeneração Tecidual da Unifesp.

ORCID: 0000-0002-6922-7719

\section{Lydia Masako Ferreira}

Médica. Pós Doc University of California, San Francisco. Profa. Titular na UNIFESP. ORCID: 0000-0003-4587-509X

Recebido em: 15/02/2021

Aprovado em: 05/04/2021
INTRODUÇÃO

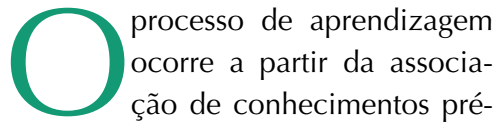
vios, que se encontram organizados na estrutura cognitiva do aluno, aos saberes adquiridos por meio de suas necessidades e potencialidades, das fragilidades individuais e da recepção de novas informações que geram novos significados. A construção de novos conhecimentos não é um processo acumulativo de informações, mas a interação do conteúdo de ensino, do método escolhido pelo professor e das condições mentais e físicas do aluno para a assimilação das teorias ${ }^{(1,2)}$.

Uma das maneiras atuais de construção e/ou transmissão do conhecimento são as Metodologias Ativas de Ensino (MAE), onde o sujeito é responsável e 
protagonista durante a aprendizagem ${ }^{(3)}$.

As MAE tornam o processo educativo dinâmico por estimular o aluno a participar de maneira intensa e com autonomia durante o processo de ensino-aprendizagem. O sucesso na utilização de MAE requer a participação e o envolvimento de todos, docentes e discentes. $\mathrm{O}$ educador deve analisar o contexto social e político do aluno para a escolha do método adequado a ser desenvolvido no contexto retratado. Entre os métodos existentes atualmente, podem ser citados: Aprendizagem Baseada em Problemas ou Projetos (Problem Based Learning), aprendizagem baseada em games e gamificação, aprendizagem entre times, sala de aula invertida, design thinking e peer instruction, estudo híbrido (blended), simuladores e tecnologias baseadas em realidade virtual, realidade aumentada e Learning Analytics, estudo de caso, Prática Baseada em Evidência (PBE), trabalho em equipe, portfólio, aprendizagem baseada na prática, pesquisa, discussão em grupo, vídeos e dramatizaçãoo ${ }^{(3-6)}$.

As MAE contribuíram para o aumento de cursos que utilizam a Educação a distância (EAD) no Brasil. Cursos disponibilizados pela rede mundial de computadores online (Internet) oferecem diversas formas de metodologias ativas de ensino e vem apresentando bons resultados, entretanto é preciso que o professor esteja atento a necessidade de motivação constante do aluno na busca constante por sua autonomia na construção do conhecimento já que a distância física entre docente e discente é uma realidade nesta modalidade de educação ${ }^{(1,2,4,7-10)}$.

Em 2017, durante o Congresso de EAD da Associação Brasileira de Educação a Distância (ABED) foi demonstrado a importância e o crescimento das MAE no Brasil, reforçando a temática de $\operatorname{EAD}^{(7,8,11,12)}$.

Conforme censo de 2017 realizado pela $\operatorname{ABED}(13)$, em 2008 havia 528.320 cursos categorizados como totalmente a distância. No ano de 2017, foram disponibilizados 1.320 .025 cursos totalmente a distância, 1.119.013 semipresenciais, 3.839.958 cursos livres não corporativos e 1.459.813 cursos livres corporativos.

Neste ano, a doença por coronavírus (COVID-19) se espalhou rapidamente pelo mundo e exigiu que os países adotassem medidas cautelares para diminuição da transmissão deste vírus. As aulas presenciais foram suspensas e foi necessário a utilização de tecnologias digitais para que os alunos não perdessem o ano letivo. A utilização de plataformas online permitiu que as aulas tradicionais se tornassem salas de aulas virtuais. Os cursos de enfermagem foram forçados a adotarem estratégias para preparar os alunos para a prática clínica ${ }^{(14-17)}$.

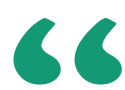

\section{Os cursos EAD facilitam o processo de formação e de atualização do indivíduo e objetivam melhorar a atuação profissional em seu dia-a-dia.}

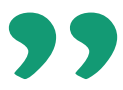

Os cursos EAD facilitam o processo de formação e de atualização do indivíduo e objetivam melhorar a atuação profissional em seu dia-a-dia. O pensamento crítico e a reflexão também devem ser estimulados na formação do aluno nos cursos EAD.

O objetivo deste estudo foi verificar e discutir as MAE adotadas na educação de enfermeiros nos cursos de capacitação EAD.

\section{MÉTODOS}

Foi realizada uma revisão integrativa de literatura para responder ao questionamento de quais MAE são utilizadas em cursos de capacitação EAD no ensino de profissionais de saúde visando caracterizar o panorama atual e perspectivas da Educação em Saúde. A pesquisa foi aprovada pelo Comitê de Ética em Pesquisa da Universidade Federal de São Paulo (UNIFESP) conforme CAAE 3988211018.

A busca da literatura ocorreu de 01 de novembro a 19 dezembro de 2020 nas bases de dados: Education Resources Information Center (ERIC), Biblioteca Virtual da Saúde (BVS) e Sistema Online de Busca e Análise de Literatura Médica (MEDLINE/ PubMed) utilizando os seguintes Descritores em Ciências da Saúde (DeCS) e suas combinações: Educação a Distância, Enfermagem e Aprendizado Ativo.

As estratégias de busca utilizadas foram: (tw:(ead or "educação a distância" or educação a distância)) AND (tw:(tecnologia da informação )) AND (tw: (Active teaching-learning methodologies)) AND (tw: (nursing education OR mh: nursing education)) e Mh: "distance education" OR distance education [tw] OR tele education [tw] OR online educations [tw] OR online education [tw].

Os critérios de inclusão foram estudos com data de publicação entre 2010 e 2020, redigidos em português, inglês ou espanhol, disponíveis gratuitamente e que descrevessem qual a MAE empregada em cursos EAD de enfermagem. Os critérios de não inclusão foram não relatar sobre a metodologia empregada e não atender aos critérios de inclusão. Os critérios de exclusão foram artigos repetidos ou que não apresentavam a ferramenta metodológica. 
Figura 1 - Estratégia para seleção dos artigos do estudo. São Paulo, 2020.

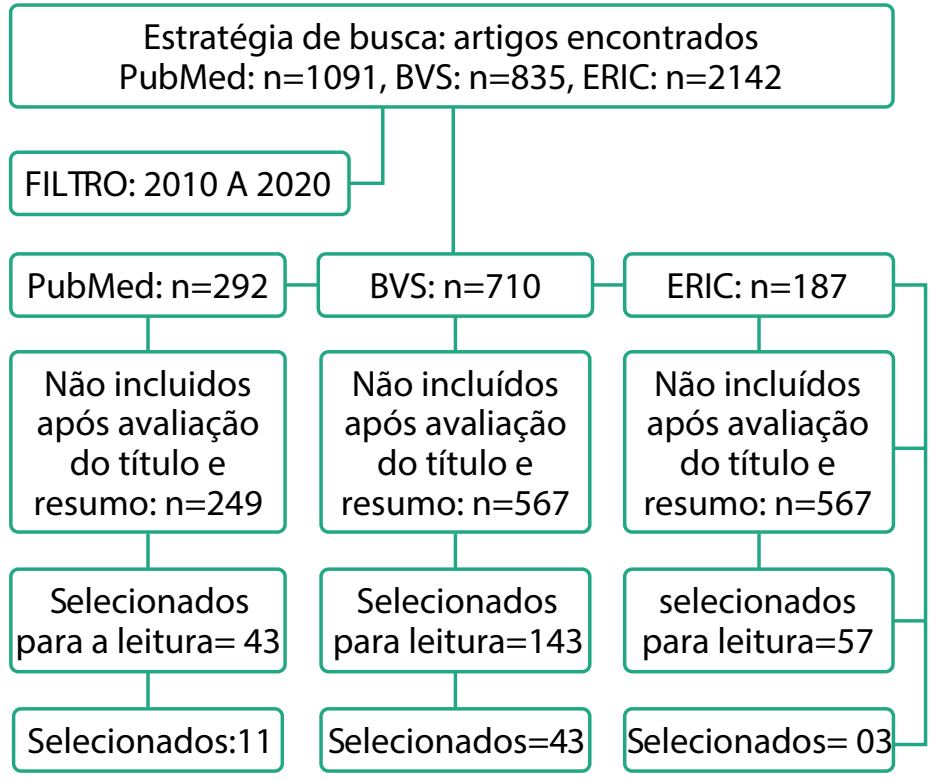

Incluídos: $\mathrm{n}=57$ Excluídos: $\mathrm{n}=186$
Após a seleção dos estudos, as MAE foram identificadas e em seguida iniciou-se o registro dos resultados.

\section{RESULTADOS}

Para esse estudo, foram selecionados e analisados 33 artigos. O fluxograma de seleção dos artigos pode ser observado na Figura 1.

Verificou-se que 20 (46,5\%) artigos tratavam de cursos com utilização do ambiente virtual de aprendizagem Moodle, $10(23,2 \%)$ realizaram e avaliaram cursos de enfermagem no formato e-learning, $6(13,9 \%)$ descreveram cursos baseados na metodologia de online learning, 5 $(11,7 \%)$ estudos retrataram o método híbrido, e 2 (4,7\%) utilizaram o aprendizado baseado na Web (Quadro 1).

As pesquisas relataram a efetividade do ensino por meio da EAD e alguns autores apontaram a necessidade de novos estudos com rigor metodológico capazes de analisar os cursos que utilizam o um ou mais MAE. Observou-se que as ferra-

Fonte: do autor

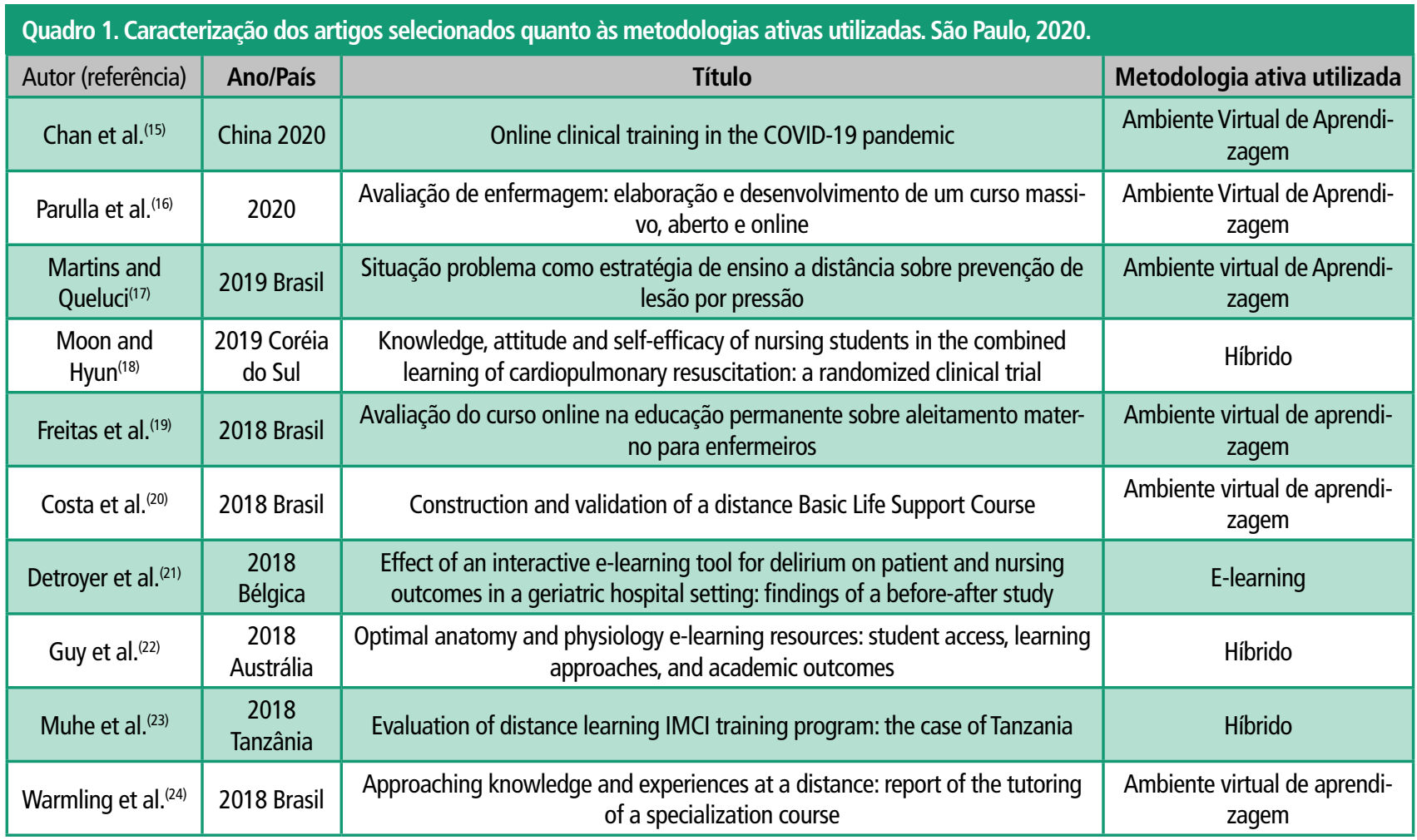




\begin{tabular}{|c|c|c|c|}
\hline Avelino et al..$^{(12)}$ & 2017 Brasil & Teaching-learning evaluation on the ICNP $®$ using virtual learning environment & $\begin{array}{l}\text { Ambiente virtual de aprendi- } \\
\text { zagem - Wiki }\end{array}$ \\
\hline Chao et al. ${ }^{(25)}$ & $\begin{array}{l}2017 \\
\text { Taiwan }\end{array}$ & $\begin{array}{l}\text { Development, implementation, and effects of an integrated web-based tea- } \\
\text { ching model in a nursing ethics course }\end{array}$ & E- learning \\
\hline $\begin{array}{l}\text { Erlandsson et } \\
\quad \text { al. }{ }^{(26)}\end{array}$ & $\begin{array}{l}2017 \\
\text { Somália }\end{array}$ & $\begin{array}{c}\text { Evaluation of an online master's programme in Somaliland. A phenomenogra- } \\
\text { phic study on the experience of professional and personal development among } \\
\text { midwifery faculty }\end{array}$ & Online learning \\
\hline Hampton et al. ${ }^{(27)}$ & 2017 EUA & $\begin{array}{l}\text { Preferred methods of learning for nursing students in an on-line degree } \\
\text { program }\end{array}$ & $\begin{array}{l}\text { E-learning Vídeos, PowerPoint } \\
\text { e estudos de caso. }\end{array}$ \\
\hline Rabeh et al. ${ }^{(28)}$ & 2017 Brasil & $\begin{array}{l}\text { Topical therapy for chronic wounds: contributions of a distance learning unit to } \\
\text { the knowledge of nursing students }\end{array}$ & $\begin{array}{l}\text { Ambiente virtual de aprendi- } \\
\text { zagem, Quiz }\end{array}$ \\
\hline Wewer et al. ${ }^{(29)}$ & $\begin{array}{l}2017 \text { Dina- } \\
\text { marca }\end{array}$ & $\begin{array}{l}\text { Health care professionals from developing countries report educational bene- } \\
\text { fits after an online diabetes course }\end{array}$ & $\begin{array}{l}\text { Curso online massivo aberto } \\
\text { (MOOC) }\end{array}$ \\
\hline Badiei et al.(1) & 2016 Iran & $\begin{array}{l}\text { Comparing nurses' knowledge retention following electronic continuous } \\
\text { education and educational booklet: a controlled trial study }\end{array}$ & E- learning \\
\hline $\begin{array}{l}\text { Pereira and } \\
\text { Sena }^{(30)}\end{array}$ & 2016 Brasil & $\begin{array}{l}\text { Courses offered by the Minas Health Channel: perception of primary care } \\
\text { workers }\end{array}$ & Plataforma Moodle (televisão) \\
\hline $\begin{array}{l}\text { Whitesman and } \\
\text { Mash }^{(31)}\end{array}$ & $\begin{array}{l}\text { 2016 África } \\
\text { do Sul }\end{array}$ & $\begin{array}{l}\text { Examining the effects of a mindfulness-based distance learning professional } \\
\text { training module on personal and professional functioning: a qualitative study }\end{array}$ & Online learning \\
\hline Xu et al. ${ }^{(32)}$ & 2016 China & $\begin{array}{l}\text { E-learning for grass-roots emergency public health personnel: preliminary } \\
\text { lessons from a national program in China. }\end{array}$ & E-learning \\
\hline Holanda et al. ${ }^{(8)}$ & 2015 Brasil & Teaching and learning in a virtual environment: nursing students' attitude & $\begin{array}{l}\text { Ambiente Virtual de Aprendi- } \\
\text { zagem: vídeos, podcast, jogos, } \\
\text { estudos de caso e fórum. }\end{array}$ \\
\hline Alves et al. ${ }^{(33)}$ & 2015 Brasil & Virtual interactivity: web forum café in a nursing management course & $\begin{array}{l}\text { Ambiente virtual de Aprendi- } \\
\text { zagem Web café }\end{array}$ \\
\hline Tronchin et al. (34) & 2015 Brasil & $\begin{array}{c}\text { Development of an online nursing management course: successful experience } \\
\text { between Brazil and Portugal }\end{array}$ & $\begin{array}{l}\text { Ambiente virtual de aprendi- } \\
\text { zagem }\end{array}$ \\
\hline Liaw et al. ${ }^{(35)}$ & $\begin{array}{l}2015 \text { Singa- } \\
\text { pura }\end{array}$ & $\begin{array}{l}\text { Designing and evaluating an interactive multimedia Web-based simulation for } \\
\text { developing nurses' competencies in acute nursing care: randomized controlled } \\
\text { trial }\end{array}$ & $\begin{array}{l}\text { Aprendizado baseada na web } \\
\text { (simulação) }\end{array}$ \\
\hline Ferreira et al. ${ }^{(36)}$ & 2015 Brasil & $\begin{array}{l}\text { Lights, camera and action in the implementation of central venous catheter } \\
\text { dressing }\end{array}$ & $\begin{array}{l}\text { Ambiente virtual de aprendi- } \\
\text { zagem (vídeo) }\end{array}$ \\
\hline Fricton et al. ${ }^{(37)}$ & 2015 EUA & $\begin{array}{l}\text { Preventing chronic pain: a human systems approach-results from a massive } \\
\text { open online course }\end{array}$ & Online Learning \\
\hline Gardiner et al. ${ }^{(38)}$ & 2015 EUA & $\begin{array}{l}\text { Online education for improving communication and documentation } \\
\text { of dietary supplements among health professionals practicing in a } \\
\text { hospital setting }\end{array}$ & Online learning \\
\hline Haugen et al. ${ }^{(39)}$ & 2015 EUA & $\begin{array}{l}\text { Harnessing technology to enhance delivery of clinical trials education for } \\
\text { nurses: a report from the children's oncology group }\end{array}$ & Online learning \\
\hline Parlakkilic ${ }^{(40)}$ & $\begin{array}{l}2015 \\
\text { Turquia }\end{array}$ & $\begin{array}{c}\text { Modular rapid e-learning framework (MORELF) in desktop virtualization } \\
\text { environment }\end{array}$ & E-learning \\
\hline Gonçalves et al. . $^{(41)}$ & 2015 Brasil & $\begin{array}{l}\text { The contribution of distance learning to the knowledge of nursing lectures } \\
\text { regarding assessment of chronic wounds }\end{array}$ & $\begin{array}{l}\text { Ambiente virtual de aprendi- } \\
\text { zagem }\end{array}$ \\
\hline Scarbrough ${ }^{(42)}$ & $\begin{array}{c}2015 \\
\text { México }\end{array}$ & $\begin{array}{l}\text { Synchronous videoconferencing in distance education for pre-licensure } \\
\text { nursing }\end{array}$ & E-learning \\
\hline $\begin{array}{l}\text { van de Steeg et } \\
\text { al.(2) }\end{array}$ & $\begin{array}{l}2014 \\
\text { Holanda }\end{array}$ & $\begin{array}{c}\text { Can an e-learning course improve nursing care for older people at risk of } \\
\text { delirium: a stepped wedge cluster randomised trial }\end{array}$ & E-learning \\
\hline
\end{tabular}




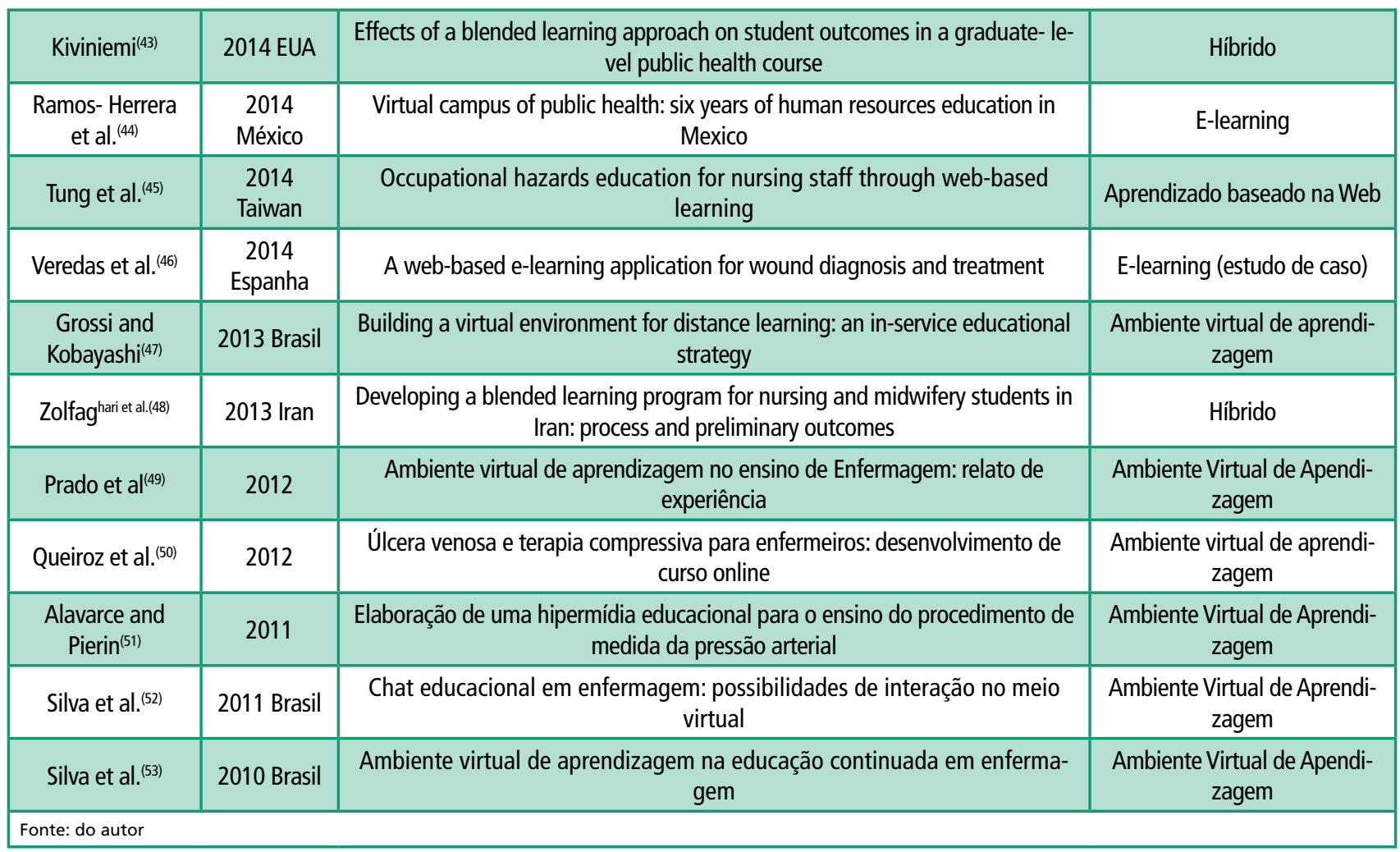

mentas metodológicas empregadas nos cursos EAD foram variadas. Como mostra o gráfico da Figura 2, 19 (58,1\%) estudos utilizaram a combinação de ferramentas tecnológicas para aplicação de MAE.

As ferramentas apresentadas na Figura 2 foram empregadas em conjunto com as seguintes MAE: aprendizagem baseada em problemas, aprendizagem baseada na prática, pesquisa, estudos de casos e discussão em grupo.

\section{DISCUSSÃO}

Para Holanda et al. ${ }^{(7,8)}$, Avelino et al. ${ }^{(12)}$, Queiroz et al. ${ }^{(50)}$, Freitas et al..(19), Alavarce et al. ${ }^{(51)}$, Silva et al. ${ }^{(21)}$, Tronchin et al. ${ }^{(28)}$, Ferreira et al. ${ }^{(30)}$ e Santos et al. ${ }^{(54)}$, o uso de tecnologias são aliadas do processo de educação continuada ${ }^{(7,8,12,34,36,54)}$. O ambiente virtual dispõe de hipertextos, links, recursos audiovisuais e fóruns de discussão que proporcionam liberdade,

Figura 2 - Recursos tecnológicos utilizados em cursos EAD para profissionais de saúde. São Paulo, 2020.

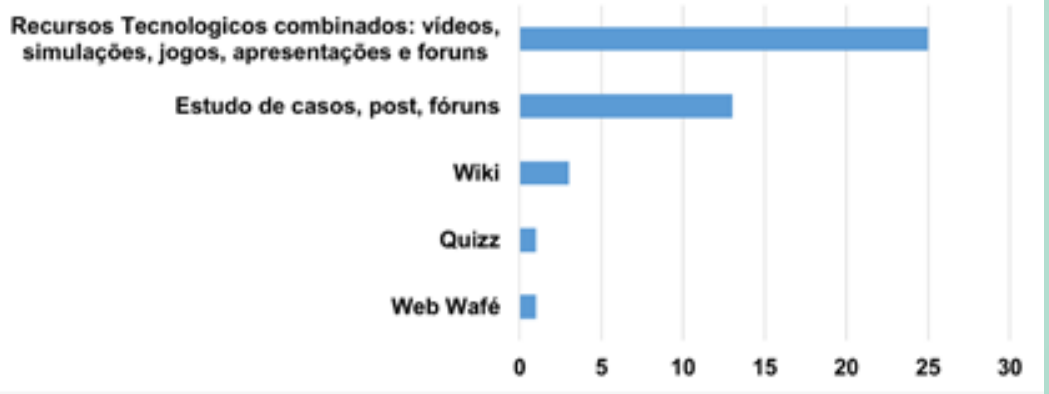

Fonte: do autor

interação e a autonomia, além de permitir ao aluno participar ativamente no seu processo de formação e/ou capacitação. Os autores reafirmaram ainda o papel fundamental dos docentes como mediadores nos cursos EAD.

Aulas online (videoaulas) e vídeos educativos são uma estratégia didática de ensino por estimular e apoiar a compreensão dos discentes em diversos contextos, tais quais: salas de aula, laboratórios e educação a distância ${ }^{(12,34,36,53,54)}$. Os vídeos contribuem para formação profissional da equipe de enfermagem por aproximar o aluno da situação clínica ainda não vivenciada, aprimoraram os conhecimentos cognitivos e habilidades técnicas relacionadas ao procedimento e são capazes de melhorar a qualidade da assistência. Outro modelo de formação são vídeos de animação que constituem uma estratégia nova e diferenciada e possibilitam a aprendizagem do aluno.

Espaços organizados com propósito didático-pedagógico criados com a utilização do ambiente virtual de 
aprendizagem Moodle apresentam bom desempenho para a implementação de atividades virtuais, com disponibilização de material didático, videoaulas sequenciais ou não, atividades acadêmicas, animações, vídeos e outras tecnologias ${ }^{(12,18,25,28,34,40,47,52,54,55)}$. O uso de videoconferências e tele consultoria como tecnologias para o auxílio de profissionais de saúde também tem se mostrado efetivo para o compartilhamento de dúvidas teóricas e da prática clínica. O chat educacional permite o desenvolvimento da autonomia no processo de construção do conhecimento dos alunos de enfermagem por meio do gerenciamento e desenvolvimento de responsabilidade pelo aprendizado e por disponibilizar outras ferramentas como: Web café, fórum e wiki (construção de textos coletivos). O ambiente virtual de aprendizagem Moodle também é definido como uma ferramenta capaz de desenvolver interatividade virtual entre os discentes de diferentes regiões e realidades, possibilitando a aprendizagem pela troca de vivencias e conhecimentos prévios que ampliam o aprendizado e a aquisição de novos conhecimentos.

Detroyer et al. ${ }^{(21)}$ utilizaram os termos e-learning e online learning para descrever o desenvolvimento de programa educacional a distância para enfermagem e van de Steeg et al. ${ }^{(2)}$ consideraram sinônimos os termos e-learning e aprendizado baseado na web, concluindo que esta abordagem educacional possui ferramentas valiosas para organizações de profissionais de saúde. De modo semelhante, Tung et al. ${ }^{(45)}$ empregaram os termos aprendizado baseado na Internet e online learning como sinônimos da metodologia aplicada no desenvolvimento de um curso sobre riscos ocupacionais.

Xu et al. ${ }^{(32)}$ utilizaram o termo e-learning para o desenvolvimento de uma plataforma com cursos digitais. O e-learning foi reconhecido como uma ferramenta educacional com as vantagens de não ter limitações de tempo e espaço, baixo custo e de fácil organização.
O termo online learning foi utilizado para descrever o desenvolvimento de um curso de mestrado que disponibilizava professores para transmissão de aulas em tempo real, reuniões virtuais semanais e atividades monitoradas na plataforma de ensino ${ }^{(26)}$. Cursos massivos online e abertos constituem ferramentas educacionais para aprendizagem online, estão hospedados em plataformas e abertos para a população com interesse na área. Os cursos possuem vídeos, fóruns de debates, entre outros recursos audiovisuais $^{(37,53)}$.

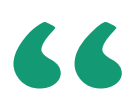

O uso de videoconferências e tele consultoria como tecnologias para o auxílio de profissionais de saúde também tem se mostrado efetivo para o compartilhamento de dúvidas teóricas e da prática clínica.

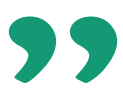

Gardiner et al. ${ }^{(38)}$ afirma que a educação online tem ganhado espaço por ser realizado de acordo com a preferência individual e fornece acesso a diversos recursos para ampliar o conhecimento.

Outros autores desenvolveram sistemas e-learning situacional interativo que integra decisões éticas em um curso para profissionais de enfermagem com o objetivo de avaliar os efeitos da capacitação na competência ética de tomada de decisão dos estudantes ${ }^{(1,25,46,56)}$. Ao final foram observados a melhora na retenção de informações, melhora do emprego do conteúdo ministrado e das atitudes e habilidades diárias relacionadas a temática.

Caveião et al. ${ }^{(5)}$, Chan et al. ${ }^{(15)}$,Prado et al. ${ }^{(49)}$, Martins et al. ${ }^{(17)}$ e Carter et al. ${ }^{(57)}$ discutem as estratégias de ensino-aprendizagem utilizadas para o desenvolvimento da liderança de enfermeiros por meio das metodologias ativas de ensino por favorecem a autonomia, despertarem a curiosidade e estimularem a tomada de decisão coletiva e individual. A MAE problem-based learning recebeu destaque por promover a autoaprendizagem e possibilitar a aplicação dos conhecimentos adquiridos na prática. O papel do docente foi o de organizar a discussão e estimular a participação de todo o grupo garantindo o aprendizado. Chan et al. ${ }^{(15)}$ utilizaram recursos como o briefing e debriefing na simulação virtual para facilitar aprendizagem do aluno. É um método eficaz para treinamento clínico, pois os alunos podem interagir com o virtual pacientes em uma recriação de um real meio ambiente.

O método híbrido foi empregado por Muhe et al. ${ }^{(23)}$, Kiviniemi ${ }^{(43)}$, Zolfaghari et al. ${ }^{(48)}$ e Moore ${ }^{(58)}$, que mesclaram aulas presenciais e online, utilizando recursos interativos e videoaulas. Considera-se que abordagens híbridas podem ser efetivas para melhorar o aprendizado e o desempenho.

Hampson et al. ${ }^{(27)}$ concluíram que a maioria dos participantes preferiram as metodologias assíncronas de ensino devido sua efetividade com a utilização de vídeos, apresentações narradas em Power Point e estudos de caso. Os participantes apontaram os vídeos e/ou apresentações em Power Point, e estudos de caso narrados como os métodos mais estimulantes e eficazes.

Os estudos encontrados demonstram a utilização de metodologias ativas combinadas no curso a distância e resultados 
positivos na aprendizagem. Apesar dos trabalhos encontrados, poucos abordam o uso de metodologia ativas de aprendizagem nos cursos de enfermagem.

\section{CONCLUSÃO}

O ano de 2020 foi acometido pela doença causada coronavírus (COVID-19), que se espalhou rapidamente pelo mundo. A adoção de medidas de contenção de transmissão da doença exigiu que os países buscassem novas alternativas para comunicação e capacitação de profissionais. A utilização de plataformas online permitiu que as aulas tradicionais se tornassem salas de aulas virtuais e assim o processo de educação a distância ganhou maior importância neste momento. Ampliou-se a discussão sobre as metodologias mais eficazes para atingir o processo de aprendizagem. No Brasil, o processo de educação está passando por mudanças didáticas e cada vez mais o aluno é estimulado a ser o protagonista no processo de ensino-aprendizagem com apoio presencial ou não de docentes responsáveis pela estruturação e acompanhamento desse processo. Neste contexto, as metodologias ativas de ensino têm sido empregadas nos cursos de formação e/ou capacitação de profissionais de enfermagem. Embora alguns

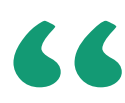

estudos apontem para o sucesso da utilização de métodos de ensino a distância são necessárias novas pesquisas com rigor metodológico sobre a temática e do desenvolvimento de técnicas de mensuração da qualidade dos cursos ofertados aos profissionais de enfermagem. Mesmo diante do desafio de educar a distância com qualidade, cabe ressaltar que a utilização das metodologias ativas de ensino nos processos de educação da enfermagem contribui para o acesso dos profissionais à informação e podem impactar positivamente, melhorando a qualidade da assistência prestada aos pacientes.

Contribuições para a área de educação em enfermagem

As Metodologias Ativas de Ensino (MAE) contribuem de forma efetiva para o processo de aprendizagem nos cursos a distância na área de educação em enfermagem com a mesma qualidade de cursos presenciais. $\mathrm{O}$ aumento de cursos a distância possibilita atingir o maior número de profissionais, impactando diretamente na qualidade de assistência prestada. A atual situação mundial com a pandemia de COVID-19 demonstrou a importância das tecnologias digitais para capacitação. 1

\section{Referências}

1. Badiei M, Gharib M, Zolfaghari M, Mojtahedzadeh R. Comparing nurses' knowledge retention following electronic continuous education and educational booklet: a controlled trial study. Med J Islam Repub Iran. 2016;30:e364. 2. van de Steeg L, IJkema R, Langelaan M, Wagner C. Can an e-learning course improve nursing care for older people at risk of delirium: a stepped wedge cluster randomised trial. BMC Geriatr. 2014;14:69.

3. Bacich M, Moran J. Metodologias ativas para uma educação inovadora: uma abordagem teórico-prática. São Paulo: Penso Editora; 2018.

4. Becerril LC, Talavera BE, Gómez BA, Rojas AM. Reflexive and critical thinking developed in nursing students: Evidence from a Mexican public university. Rev Uruguaya Enferm. 2017;12(1):1-19.

5. Caveião C, Peres AM, Zagonel IP, Amestoy SC, Meier MJ. Teaching-learning tendencies and strategies used in the leadership development of nurses. Rev Bras Enferm. 2018;71(suppl 4):1622-30.

6. Pascon DM, Otrenti E, Mira VL. Percepção e desempenho de graduandos de enfermagem em avaliação de metodologias ativas. Acta Paul Enferm. 2018;31(1):61-70.

7. Holanda VR, Pinheiro AK, Pagliuca LM. Learning in online education: analysis of concept. Rev Bras Enferm. 2013;66(3):406-11.

8. Holanda VR, Pinheiro AK, Holanda ER, Santos MC. Teaching and learning in a virtual environment: nursing students' attitude. Rev Min Enferm.
2015;19(1):141-7.

9. Dellaroza MS, Tada CN, Haddad MC; Vannuchi MT, Maziero VG. The teaching of nursing management in undergraduate: an integrative review. Semina Cienc Biol Saude. 2015;36(1):149-58.

10. Silva AP, Pedro EN. Autonomy in nursing students' process of knowledge construction: the educational chat as a teaching tool. Rev Latino-Am Enferm. 2010;18(2):73-80.

11. Yuksekdag BB, Barlas GU. The atitude scale towards distance nursing education (astDNE). Turkish Online J Distance Educ. 2015;16(1):52-61.

12. Avelino CC, Costa LC, Buchhorn SM, Nogueira DA, Goyatá SL. Teaching-learning evaluation on the ICNP® using virtual learning environment. Rev Bras Enferm. 2017;70(3):630-7.

13. Associação Brasileira de Educação a Distância (ABED). 2017 Brazilian census for distance learning: analytic report of distance learning in Brazil. Curitiba: Editora InterSaberes; 2018.

14. Yuet-Ming Ng, Pui Lai P. Coronavirus disease (COVID-19) prevention: Virtual classroom education for hand hygiene. J Nurse Educ Pract. 2020;45:1-2. 15. Chan MMK, Yu DSF, Lam VSF, Wong JYH. Online clinical training in the COVID-19 pandemic () 2020. J Clin Teach.2020;17:1-2.

16. Parullaa CD, Galdino DM, Paic DD, Azzolinc KO, Cogoc ALP. Nursing assessment: the elaboration and development of a massive open online course. Rev 


\section{Referências}

Gaúcha Enferm. 2020;41(esp):1-5.

17. Martins CLM, Queluci GC. Situation resolution as a distance teaching strategy on pressure injury prevention. Rev Enferm Foco. 2019;10(6):99-104. 18. Moon H, Hyun HS. Knowledge, attitude and self-efficacy of nursing students in the combined learning of cardiopulmonary resuscitation: a randomized clinical trial. BMC Med Educ. 2019;19:414.

19. Freitas LA, Costa LCS, Costa AS, Avelino CCV, Ribeiro PM, Goyatá SLT. Avaliação do curso on line de educação permanente sobre aleitamento materno para enfermeiros. Rev Enferm UFSM. 2018;8(1):116-28.

20. Costa IK, Tibúrcio MP, Melo GS, Leite JE, Dantas RA, Torres GV. Construction and validation of a distance Basic Life Support Course. Rev Bras Enferm. 2018;71(suppl 6):2698-705.

21. Detroyer E, Dobbels F, Teodorczuk A, Deschodt M, Depaifve $Y$, Joosten $E$, et al. Effect of an interactive e-learning tool for delirium on patient and nursing outcomes in a geriatric hospital setting: findings of a before-after study. BMC Geriatr. 2018;18(1):19.

22. Guy R, Byrne B, Dobos M. Optional anatomy and physiology e-learning resources: student access, learning approaches, and academic outcomes. Adv Physiol Educ. 2018;42(1):43-9.

23. Muhe LM, Iriya N, Bundala F, Azayo M, Bakari MJ, Hussein A, et al. Evaluation of distance learning IMCI training program: the case of Tanzania. BMC Health Serv Res. 2018;18(1):547.

24. Warmling D, Boell JEW, Costa VT, Peres GM, Faust SB, Bolsoni CC, et al. Approaching knowledge and experiences at a distance: report of the tutoring of a specialization course. Rev Salud Publica. 2018;20(1):132-7.

25. Chao SY, Chang YC, Yang SC, Clark MJ. Development, implementation, and effects of an integrated web-based teaching model in a nursing ethics course. Nursing Educ Today. 2017;55:31-7.

26. Erlandsson K, Osman F, Hatakka M, Egal JA, Byrskog U, Pedersen C, et al. Evaluation of an online master's programme in Somaliland. A phenomenographic study on the experience of professional and personal development among midwifery faculty. Nurse Educ Pract. 2017;25:96-103.

27. Hampton D, Pearce PF, Moser DK. Preferred methods of learning for nursing students in an on-line degree program. J Prof Nurs. 2017;33(1):27-37.

28. Rabeh SA, Gonçalves MB, Caliri MH, Nogueira PC, Miyazaki MY. Topical therapy for chronic wounds: contributions of a distance learning unit to the knowledge of nursing students. Enferm Glob. 2017;45:80-90.

29. Wewer ANJ, Poulsen KW, Svensson Lø, Jensen L, Holst JJ, Torekov SS. Health care professionals from developing countries report educational benefits after an online diabetes course. BMC Med Educ. 2017;17(1):97.

30. Pereira LD, Sena RR. Courses offered by the Minas Health Channel: perception of primary care workers. Rev Gaucha Enferm. 2016:37(2):e56984.

31. Whitesman S, Mash R. Examining the effects of a mindfulness-based distance learning professional training module on personal and professional functioning: a qualitative study. BMC Med Educ. 2016;16(1):287.

32. Xu W, Jiang Q, Qin X, Fang G, Hu Z. E-learning for grass-roots emergency public health personnel: preliminary lessons from a national program in China. Biosci Trends. 2016;10(3):235-9.

33. Alves VL, Okagawa FS, Parra JF, Bohomol E, Cunha IC. Virtual interactivity: web forum café in a nursing management course. Rev Min Enferm. 2015;19(1):127-33

34. Tronchin DM, Peres HH, Lima AF, Alavarce DC, Prata AP, Santos MR, et al. Development of an online nursing management course: successful experience between Brazil and Portugal. Rev Esc Enferm USP. 2015;49(Sp 2):162-7.

35. Liaw SY, Wong LF, Chan SW, Ho JT, Mordiffi SZ, Ang SB, et al. Designing and evaluating an interactive multimedia Web-based simulation for developing nurses' competencies in acute nursing care: randomized controlled trial. J Med Internet Res. 2015;17(1):e5.

36. Ferreira MV, Godoy S, Góes FS, Rossini FP, Andrade D. Lights, camera and action in the implementation of central venous catheter dressing. Rev Latino-Am Enferm. 2015;23(6):1181-6.

37. Fricton J, Anderson K, Clavel A, Fricton R, Hathaway K, Kang W, et al. Preventing chronic pain: a human systems approach-results from a massive open online course. Glob Adv Health Med. 2015;4(5):23-32.

38. Gardiner P, Filippelli AC, Kabbara K, Lin SC, Sadikova E, Kaptchuk TJ, et al. online education for improving communication and documentation of dietary supplements among health professionals practicing in a hospital setting. J Altern Complement Med. 2015;21(10):638-44.

39. Haugen $M$, Gasber $E$, Leonard $M$, Landier W. Harnessing technology to enhance delivery of clinical trials education for nurses: a report from the Children's Oncology Group. J Pediatr Oncol Nurs. 2015;32(2):96-102.

40. Parlakkilic A. Modular rapid e-learning framework (MORELF) in desktop virtualization environment. Turkish Online J Distance Educ. 2015;16(1):1-16.

41. Gonçalves MB, Rabeh SA, Terçariol CA. The contribution of distance learning to the knowledge of nursing lecturers regarding assessment of chronic wounds. Rev Latino-Am Enferm. 2015;23(1):122-9.

42. Scarbrough JE. Synchronous videoconferencing in distance education for pre-licensure nursing. J Educ Training Stud. 2015;3(4):62-72.

43. Kiviniemi MT. Effects of a blended learning approach on student outcomes in a graduate-level public health course. BMC Med Educ. 2014;14:47.

44. Ramos-Herrera I, Alfaro-Alfaro N, Fonseca-León J, García-Sandoval C, González-Castañeda M, López-Zermeño Mdel C, et al. Virtual campus of public health: six years of human resources education in Mexico. Rev Panam Salud Publica. 2014:36(5):342-7.

45. Tung CY, Chang CC, Ming JL, Chao KP. Occupational hazards education for nursing staff through web-based learning. Int J Environ Res Public Health. 2014;11(12):13035-46.

46. Veredas FJ, Ruiz-Bandera E, Villa-Estrada F, Rufino-González JF, Morente L. A web-based e-learning application for wound diagnosis and treatment. Comput Methods Programs Biomed. 2014;116(3):236-48.

47. Grossi MG, Kobayashi RM. Building a virtual environment for distance learning: an in-service educational strategy. Rev Esc Enferm USP. 2013;47(3):756-60.

48. Zolfaghari M, Negarandeh R, Eybpoosh S. Developing a blended learning program for nursing and midwifery students in Iran: process and preliminary outcomes. Iran J Nurs Midwifery Res. 2013;18(1):20-6.

49. Prado C, Santiago LC, Silva JAM, Pereira IM, Leonello VM, Otrenti E, et al. Ambiente virtual de aprendizagem no ensino de Enfermagem: relato de experiência. Rev Bras Enferm. 2012;65(5):862-6.

50. Queiroz FM, Aroldi JBC, Oliveira GDS, Peres HHC, Santos VLCG. Venous ulcer and compression therapy for nurses: development of online course. Rev Acta Paul Enferm. 2012;25(3):435-40.

51. Alavarce DC, Pierin AMG. Elaboração de uma hipermídia educacional para o ensino do procedimento de medida da pressão arterial. Rev Esc Enferm. 2011; 45(4):939-44.

52. Silva APSS, Pedro ENR, Cogo ALP. Chat educacional em enfermagem: possibilidades de interação no meio virtual. Rev Esc Enferm. 2011;45(5):1213-20. 53. Silva LMG, Gutiérrez MGR, Domenico EBL. Ambiente virtual de aprendizagem na educação continuada em enfermagem. Acta Paul Enferm. 2010;23(5):701-4.

54. Santos JL, Souza CS, Tourinho FS, Sebold LF, Kempfer SS, Linch GF. Didactic strategies in the teaching-learning process of nursing management. Texto Contexto Enferm. 2018;27(2):1-11.

55. Tavares AP, Leite BS, Silveira IA, Santos TD, Brito WA, Camacho AC. Analysis of Brazilian publications on distance education in nursing: integrative review. Rev Bras Enferm. 2018;71(1):214-22.

56. Ofoha D, Iwuchukwu 0. Employers' perception and expectations of professional competency of distance learning graduates: a tracer study of nursing graduates of the National Open University of Nigeria (NOUN). Open Praxis. 2018;10(3):265-78.

57. Carter L, Hanna M, Warry W. Perceptions of the impact of online learning as a distance-based learning model on the professional practices of working nurses in northern Ontario. Canadian J Learn Technol. 2016;42(3):1-15. 58. Moore ZKAB, Auliffe F. Virtual adaptation of traditional healthcare quality improvement trainingin response to COVID-19: a rapid narrative review. Hum Resour Health. 2020;18(81):1-16. 